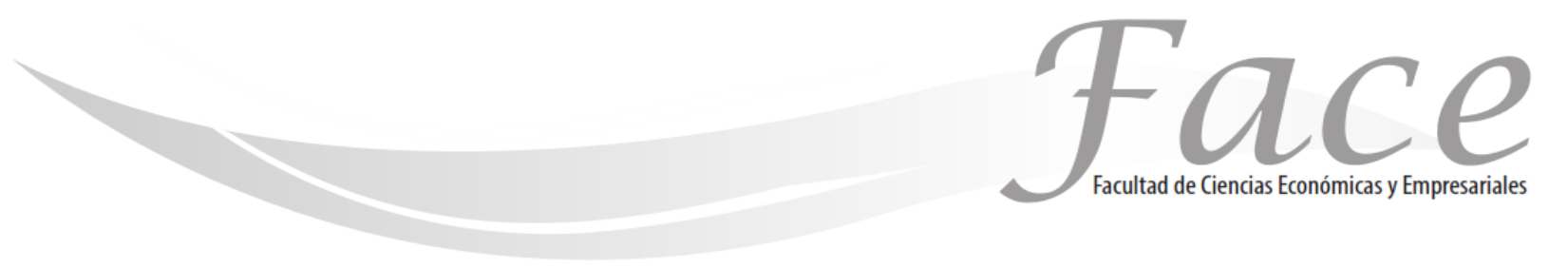

ISSN Impreso: 1794-9920 ISSN Electrónico: 2500-9338

Volumen $17-\mathrm{N}^{\circ} 2$

Año 2017

Págs. 157 - 173

\title{
BENCHMARKING Y ANÁLISIS DE COMPETITIVIDAD DE LAS CADENAS PRODUCTIVAS DE QUINUA EN COLOMBIA, PERU Y BOLIVIA *
}

\author{
Jahir Lombana ** \\ Enlace ORCID: https://orcid.org/0000-0002-0055-8392 \\ **Yasser Amashta *** \\ Enlace https://orcid.org/0000-0002-2944-3468 \\ ${ }^{* * *}$ Carolina Correa **** \\ Enlace ORCID:https://orcid.org/0000-0002-4344-2840 \\ **** Maria-Carolina Rodríguez ***** \\ Enlace ORCID:https://orcid.org/0000-0002-6625-8210
}

Fecha de Recepción: 1 de junio 2017

Fecha de Aprobación: 20 de Julio 2017

\section{Resumen:}

Para sugerir a la quinua como producto de calidad con sello colombiano, este estudio tiene como objetivo hacer el benchmarking de producción/comercialización colombiana de quinua frente a las cadenas productivas de Bolivia y Perú (referentes mundiales en producción y exportaciones) a través del diamante de la competitividad de Porter. Se concluye Bolivia sigue siendo importante pero su productividad a limitado su crecimiento, en tanto su producción sigue dependiendo de bajos costos de mano de obra. En cambio, el desarrollo creciente de Perú en el sector puede servir de ejemplo para Colombia.

Palabras clave: cadena productiva, Diamante de Porter, competitividad, benchmarking, quinua

* El artículo es subproducto de un trabajo de grado para optar al título de maestría en Negocios Internacionales de la Universidad del NorteColombia.

** Ph.D. Docente/Investigador, Escuela de Negocios Universidad del Norte, Barranquilla - Colombia. Correo: lombanaj@uninorte.edu.co

*** Máster en Negocios Internacionales, Escuela de Negocios Universidad del Norte, Barranquilla - Colombia. Correo: jamashta@gmail.com

**** Máster en Negocios Internacionales, Escuela de Negocios Universidad del Norte, Barranquilla - Colombia. Correo: carocorreagallardo@gmail.com

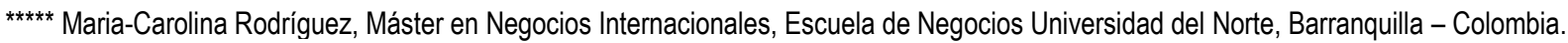
Correo: carorodriguez53@gmail.com 


\title{
BENCHMARKING AND ANALYSIS OF COMPETITIVENESS OF QUINUA PRODUCTIVE CHAINS IN COLOMBIA, PERU AND BOLIVIA
}

\begin{abstract}
In order to suggest quinoa as a quality product with Colombian seal, this study aims to benchmark Colombian quinoa production/commercialization in relation to the production chains of Bolivia and Peru (worldwide referents in production and exports) through the diamond of the competitiveness of Porter. It is concluded that Bolivia continues to be important but its productivity has limited its growth, while its production continues to depend on low labor costs. Instead, Peru's growing development in the sector can serve as an example for Colombia.
\end{abstract}

Keywords: Productive chain, Porter's diamond, competitiveness, benchmarking, quinoa

\section{BENCHMARKING E ANÁLISE DA COMPETITIVIDADE DAS CADEIAS PRODUTIVAS QUINUA NA COLÔMBIA, NO PERU E NA BOLÍVIA}

\section{Resumo}

\section{Resumo:}

Para sugerir quinoa como um produto de qualidade com um selo colombiano, este estudo tem como objetivo comparar a produção / comercialização colombiana de quinoa em relação às cadeias produtivas da Bolívia e do Peru (referência mundial em produção e exportação) através do diamante de a competitividade de Porter. Conclui que a Bolívia continua sendo importante, mas sua produtividade limitou seu crescimento, enquanto a sua produção continua a depender de baixos custos trabalhistas. Por outro lado, o crescente desenvolvimento peruano no setor pode servir de exemplo para a Colômbia.

Palavras-chave: cadeia produtiva, diamante Porter, competitividade, benchmarking, quinoa 


\section{INTRODUCCIÓN:}

Con el aumento de la población mundial y la escasez de recursos para alimentar de una forma digna a todas las personas, organizaciones como las Naciones Unidas para la Alimentación y Agricultura (FAO - Food and Agriculture Organization of the United Nations) han estado buscando formas para garantizar la seguridad alimentaria mundial. Entre las alternativas propuestas se ha hecho hincapié en un producto que a pesar de ser de tiempos pre-colombinos en países andinos, es relativamente nuevo para el resto del mundo: la quinua.

En el documento de antecedentes sobre la quinua, la FAO (2011) hace un recorrido sobre las características especiales de esta semilla. La quinua es una planta originaria de la América Andina y que se cultiva desde épocas precolombinas. Su tamaño oscila entre los $0.5-2 \mathrm{~m}$ de altura, posee un tallo recto o ramificado y su color es variable. Las semillas, que constituyen la parte de mayor valor alimenticio, son pequeños gránulos con diámetros de entre 1.8 y $2.2 \mathrm{~mm}$.

La quinua tiene una extraordinaria adaptabilidad a climas diversos: desde el desértico hasta climas calurosos y secos, puede crecer con humedades relativas desde $40 \%$ hasta $88 \%$, y soporta temperaturas desde $-4^{\circ} \mathrm{C}$ hasta $38^{\circ} \mathrm{C}$. Es una planta eficiente al uso de agua, es tolerante y resistente a la falta de humedad del suelo, y permite producciones aceptables con precipitaciones de 100 a $200 \mathrm{~mm}$.

Existen muchos beneficios del cultivo de la quinua, iniciando con su alto valor nutricional debido a que gracias al elevado contenido de aminoácidos esenciales de su proteína, la quinua es considerada como el único alimento del reino vegetal que provee todos los aminoácidos esenciales. Además del alto contenido proteico, provee grasas, fibra, antioxidantes, minerales, vitaminas y es libre de gluten. Así, a partir de la quinua se puede obtener una serie de subproductos de uso medicinal, cosmético e industrial.

Este grano tiene una cantidad de características (nutritivas y organolépticas) que lo hacen ideal como medida paliativa para los altos niveles de hambre mundiales. Con el interés mostrado por la FAO $(2011,2013$ \& 2014), el mundo ha volcado su mirada a este ancestral alimento, y su demanda ha ido aumentando. Muchos países han tomado ventaja de esta situación y han asegurado su posición en el mercado internacional, entre estos están Bolivia y Perú (FAO 2014)., que son los mayores productores de quinua a nivel mundial. Colombia como país andino también goza de iguales ventajas geográficas, sin embargo su producción aún es incipiente.

Aunque es cierto que existen pruebas a nivel internacional de producción del grano en otros países como en Estados Unidos y Canadá (Murphy \& Matanguihan, 2015), India (Bhargava \& Ohri, 2014), Mali (Coulibaly et al, 2014) y Europa (Jacobsen, 1997 \& Piva, Brasse y Mehinagic 2013), la ventaja comparativa por ubicación geográfica coloca a los países productores andinos en los niveles de mayor productividad (Winkel et al, 2012).

Bolivia se mantuvo como el primer productor y exportador de quinua en el mundo hasta 2014, año en el cual fue superado por Perú (Ministerio Agricultura Perú, 2015 y 2017). El caso peruano llama la atención, ya que ha aumentado su participación del 6\% en el intervalo de 1992 a 1996 hasta un 23\% entre 2008 y 2012 (FAO, 2014), esto ha resultado por la productividad de Perú en los últimos años.

La falta de conocimiento y difusión, así como de reglamentación por parte del gobierno colombiano del cultivo de la quinua han retrasado su desarrollo no existiendo una producción uniforme en las áreas sembradas (Jäger, 2015a). Para poder remediar la precaria situación de Colombia se sugiere comprender el panorama de las cadenas de producción al interior de países como Perú y Bolivia, y realizar una comparación completa de los aspectos de tales cadenas en los respectivos países. Una vez se establezcan las ventajas y desventajas competitivas de cada cadena, se podrán generar propuestas para el mejoramiento de la producción de quinua en Colombia. Es así como el objetivo de este documento es presentar un análisis de benchmarking y competitivo de las cadenas productivas referentes de Perú y Bolivia que serán comparadas con Colombia para establecer algunas recomendaciones de política. Después de esta introducción se presenta el marco teórico para el análisis teniendo en cuenta el concepto de competitividad que usará el modelo de diamante de Porter (1991) en las cadena productivas de los países seleccionados para ser comparados de acuerdo a la metodología del benchmarking. Luego se explica la metodología que es fundamentalmente cualitativa y de enfoque descriptivo. Los resultados se presentan para los tres países y se cierra el documento con las conclusiones. 


\section{MARCO TEÓRICO:}

Existen diferentes apreciaciones del concepto de competitividad con respecto a la unidad de análisis en la que se esté manejando, háblese de empresas, instituciones y organizaciones en un nivel micro, y naciones en un nivel macro (Lombana \& Rozas, 2009). Es complejo llegar a una definición única de competitividad, más teniendo en cuenta las diferentes posiciones que se tienen al respecto y sus elementos críticos de que cabe todo (Krugman, 1994 \& 2005). Quizás los mayores consensos se encuentren en que competitividad incorpora una dinámica de las unidades de análisis y su comparación frente a un referente y dependerá entonces del investigador acogerse a una definición que le sea funcional a sus propósitos (Lombana 2006). Para el caso de este trabajo con énfasis en el sector agrícola Rojas y Sepúlveda (1999, p. 18), plantean la siguiente definición que recoge varios puntos clave para la investigación:

"[...] Competitividad es un concepto comparativo fundamentado en la capacidad dinámica que tiene una cadena agroalimentaria localizada espacialmente, para mantener, ampliar y mejorar de manera continua y sostenida su participación en el mercado, tanto doméstico como extranjero, a través de la producción, distribución y venta de bienes y servicios en el tiempo, lugar y forma solicitados, buscando como fin último el beneficio de la sociedad.

Tal capacidad depende de una serie de elementos a nivel macro, meso y micro, tanto económicos como no económicos. A nivel macro intervienen aspectos referidos al país y a sus relaciones con el resto del mundo. A nivel meso se destacan factores espaciales: distancia, infraestructura de apoyo a la producción, base de recursos naturales e infraestructura social. En el nivel micro, se destacan los factores relevantes para la empresa, referidos a precio y calidad, así como factores espaciales que condicionan directamente a la empresa [...]"
Como se puede apreciar los autores hacen énfasis en cadenas agroalimentarias y participación de mercado, que son precisamente dos aspectos importantes para analizar en este estudio.

El modelo teórico de Porter (1991) denominado como el diamante de la competitividad, permite de manera funcional ubicar los determinantes de la competitividad sectorial (ver gráfico 1) que precisamente acuña con su teoría de la ventaja competitiva y que implica un proceso de innovación constante. Esto es, no depender solamente de la ventaja que se tenga con respecto a algún factor productivo (capital, tierra, trabajo), sino a ir más allá para lograr una diferenciación con respecto a los otros competidores, pero además, apuntar a una mejoría constante para sostener dicha ventaja a largo plazo.

Tener una ventaja competitiva implica: determinar un potencial no explotado para desarrollar, lograr una diferenciación del resto de los competidores, y mediante un proceso de innovación persistente usando el factor tecnológico, sostener la competitividad en el largo plazo.

El modelo de Porter no está exento de críticas particularmente cuando es aplicado a países en desarrollo y supone un mundo totalmente liberal.

Dunning (1993) y Rugman y Verbeke (1993) criticaban la posición de Porter en tanto las empresas particularmente en países en desarrollo se adaptaban y el gobierno influenciaba y era influenciado, esto contrario a la posición liberal de Porter. Por su parte estudios sobre estrategia empresarial como Hoskisson, Eden, Lau y Wright (2000) y Wright, Filatotchev, Hoskisson y Peng (2005) hacen énfasis precisamente sobre esas limitaciones. 


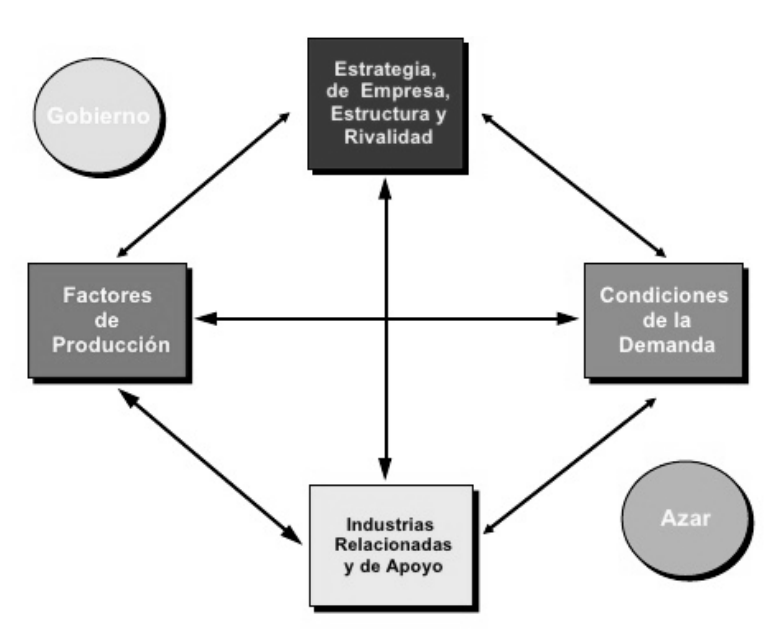

Fuente: Porter (1991)

Porter usa este diamante para determinar qué empresas e industrias tienen ventajas competitivas, y hace énfasis en la importancia de las industrias relacionadas y de apoyo donde se puede agregar a este marco el concepto de cadena productiva que se consolida en la década de los ochenta con una visión holista de la producción agroalimentaria. La teoría de sistemas y los agronegocios ampliaron el concepto de sistema de finca agrícola añadiendo agentes antes y después de la actividad productiva (Gomes de Castro, ValleLima y Pedroso 2002). El primer modelo general de cadena productiva agrícola propuesto por Gomes de Castro, ValleLima \& Freitas en 1988 se constituyó por eslabones que representaron a proveedores de insumos, fincas, agroindustrias, comercializadoras y consumidores integrados e interconectados por flujos de capital, de materiales y de información. Nuevamente con Porter y Millar (1985) este concepto de cadena productiva puede ampliarse al de cadena de valor que además de unir los eslabones verticales de productor a consumidor remite a encadenamientos horizontales con empresas que cooperan y rivalizan a manera de clústeres (Cifuentes, Pérez, y GilCasares 2011).

Es en un modelo ampliado de competitividad donde resulta necesario incorporar a los competidores que serán los referentes del estudio. En algunos casos las empresas no son las más competitivas y ven en sus pares posibilidades de desarrollo. El análisis de benchmark puede ser útil para este caso y su uso y utilidad ha sido documentado a través de la historia por varios autores, entre ellos McNair y Leibfried (1992), Spendolini (1992), Watson (1993) y Boxwell (1994); pero la conceptualización formal se le atribuye a Camp (1989), quien aplicó la herramienta a la empresa Xerox y basado en esta experiencia publicó su obra. Camp refiere en su tipología de benchmark a: 1) identificación de las mejores prácticas; 2) identificación de tendencias o 3) comparar experiencias. Es precisamente en este último tipo al que se refiere este estudio. Esta herramienta de análisis estratégico está en constante evolución como se confirma por sus innovaciones teóricas acerca de su contenido, tipología, ámbito de aplicación y objetivos (Gurutze \& Ochoa, 2005, Fernandez, Mccarthy \& Rakotobe-Joel 2001, Kyrö, 2003).

Adentrándose en la unidad de análisis de este estudio, la quinua a pesar de ser un cultivo con milenios de tradición, sólo ha experimentado un nivel de atención elevado en los últimos años. Se da un énfasis especial cuando la ONU declaró el año 2013 como el año de la quinua, lo que ha promovido diferentes estudios tanto gubernamentales como privados, para dar luz al panorama de la producción y mercado de la quinua.

Entre tales estudios se encuentran varios informes entregados por la FAO, acerca de las características nutricionales de la quinua y el proceso de producción en países andinos donde es más popular, como son: Quinua: Cultivo milenario para contribuir a la seguridad alimentaria mundial, publicado en 2011 donde se describen las características de la quinua y su producción, y que se complementa con Quinua: Operaciones de Poscosecha, publicado en 2013, que detalla el proceso de producción de la quinua. Los temas comerciales son más ampliamente tratados en los documentos Tendencias y Perspectivas del Comercio Internacional de Quinua, realizado en conjunto con ALADI y publicado en el año 2014, que generalizan acerca del panorama en años venideros del mercado de la quinua; y por último, Reporte deL Estado del Arte de la Quinua alrededor del mundo en 2013, publicado en 2014, el cual es un compendio general de panorama de la quinua a nivel mundial. 
También existen otros informes por país como el del Instituto Interamericano de Cooperación para la Agricultura, IICA, que en 2015 publica sendos reportes El Mercado y producción de quinua en el Perú, y el de Producción y Mercado de la Quinua en Bolivia (Risi, Rojas y Pacheco,

2015) donde se da una aproximación a los procesos de producción y exportación de quinua desde estos países.

Para Colombia, se empieza a notar además del interés comercial, el académico, cuando en marzo de 2015 se realiza un taller en la ciudad de Cali (Colombia) El cultivo de la quinua en Colombia y sus perspectivas futuras, en el cual se reunieron los principales actores de la cadena de producción de la quinua en el país para determinar el panorama actual, las falencias presentadas y estrategias para mitigar estas falencias (Jägger, 2015b). Existen también aproximaciones desde el punto de vista comercial en Cely-Torres y Ducon-Salas (2015) y Montoya Martínez y Peralta (2005), aunque ninguna de estas en perspectiva comparada.

Es creciente el interés por la quinua y este estudio presenta un punto de partida por su interés comercial desde Colombia, aprendiendo sobre el camino ya recorrido por los países de referencia.

\section{METODOLOGÍA:}

La presente investigación es de carácter cualitativo descriptivo, en tanto recopila información de diversas fuentes secundarias para conocer la naturaleza del problema, su realidad contextual, evolución, y establecer comparaciones que puedan tomarse de base para la generación de estrategias de cambio y mejoramiento de resultados. Esto implica una revisión sistemática de documentos con información pertinente a la competitividad de la cadena productiva de la quinua en Colombia, Perú y Bolivia.

Asimismo se realizaron visitas de campo y entrevistas a actores de la cadena de producción de quinua en el
Departamento del Cauca, Colombia. Debido a dificultades de desplazamiento hacia Perú y Bolivia, no se aplicaron cuestionarios a sus productores 0 entidades pero tanto productores y entidades relacionadas con el sector en Colombia ya se habían aproximado a los países productores seleccionados y conocían de primera mano su situación, esto permitió validar lo encontrado en fuentes secundarias

de dichos países. Una vez realizada la compilación de información primaria y secundaria se realiza el análisis estratégico utilizando el modelo del diamante de Porter (1991) y las referencias citadas del benchmarking, esto lleva a encontrar la interacción entre las características particulares y su entorno a cada país en las respectivas cadenas productivas de quinua, primero estableciendo sus ventajas y desventajas y luego sus factores claves de éxito y podrá servir como base para la generación de estrategias para el caso colombiano.

\section{RESULTADOS Y DISCUSIÓN:}

En los últimos años, se constata un progresivo aumento de la producción de quinua, especialmente en los países que han sido tradicionalmente los principales productores, esto es Bolivia, Perú y Ecuador, y se estima que más del $80 \%$ de la producción mundial de quinua se concentra en esos tres países (FAO - ALADI, 2014).

Es de destacar que la producción de Bolivia ha sido creciente particularmente desde el año 2011, sin embargo en términos de productividad, el Perú durante la primera década del 2000 siempre supero a Bolivia con $1100 \mathrm{kgs} / \mathrm{ha}$. Es en 2014 cuando este valor llega a los $1683 \mathrm{kgs} / \mathrm{ha}$ coincidiendo con la reducción de la producción boliviana. Cabe anotar que desde 2003, la productividad del Ecuador también ha superado a la de Bolivia. Esto muestra de que a pesar de su gran participación en el mercado, en términos de productividad se ha quedado estancado, facilitando el acceso a nuevos actores en producción (ver gráfico 2). 
Gráfico $\mathbf{N}^{\circ} 2$.

Área cultivada y productividad

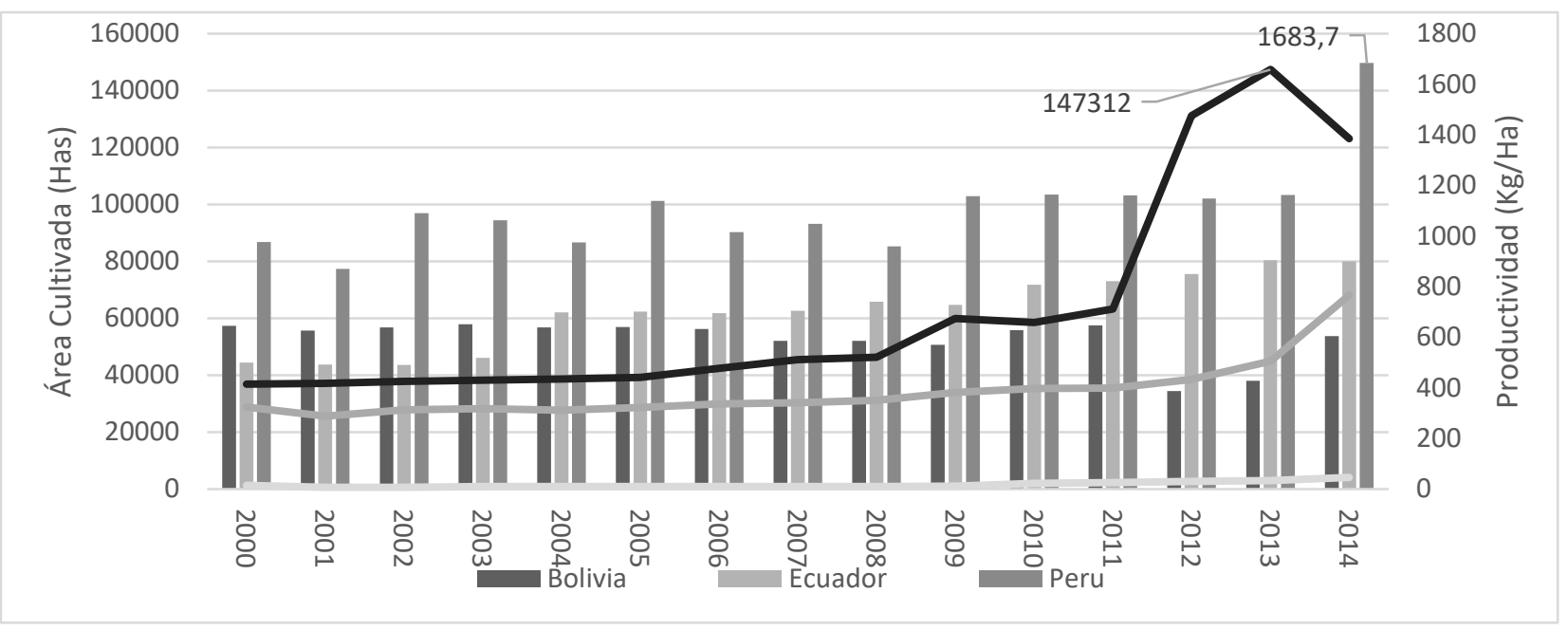

Fuente: Elaboración propia con información de FAOSTAT (2017)

Históricamente, Bolivia había sido el mayor productor de quinua, seguido de Perú. Sin embargo, esto cambió en 2014 cuando Perú sobrepasó a Bolivia y se ubica desde entonces como el mayor productor de quinua. Ecuador es el tercer país productor de quinua, aunque a distancia apreciable de Perú y Bolivia (ver gráfico 3).

Gráfico $\mathbf{N}^{\circ} 3$.

Producción y valor de quinua en Bolivia, Perú y Ecuador.

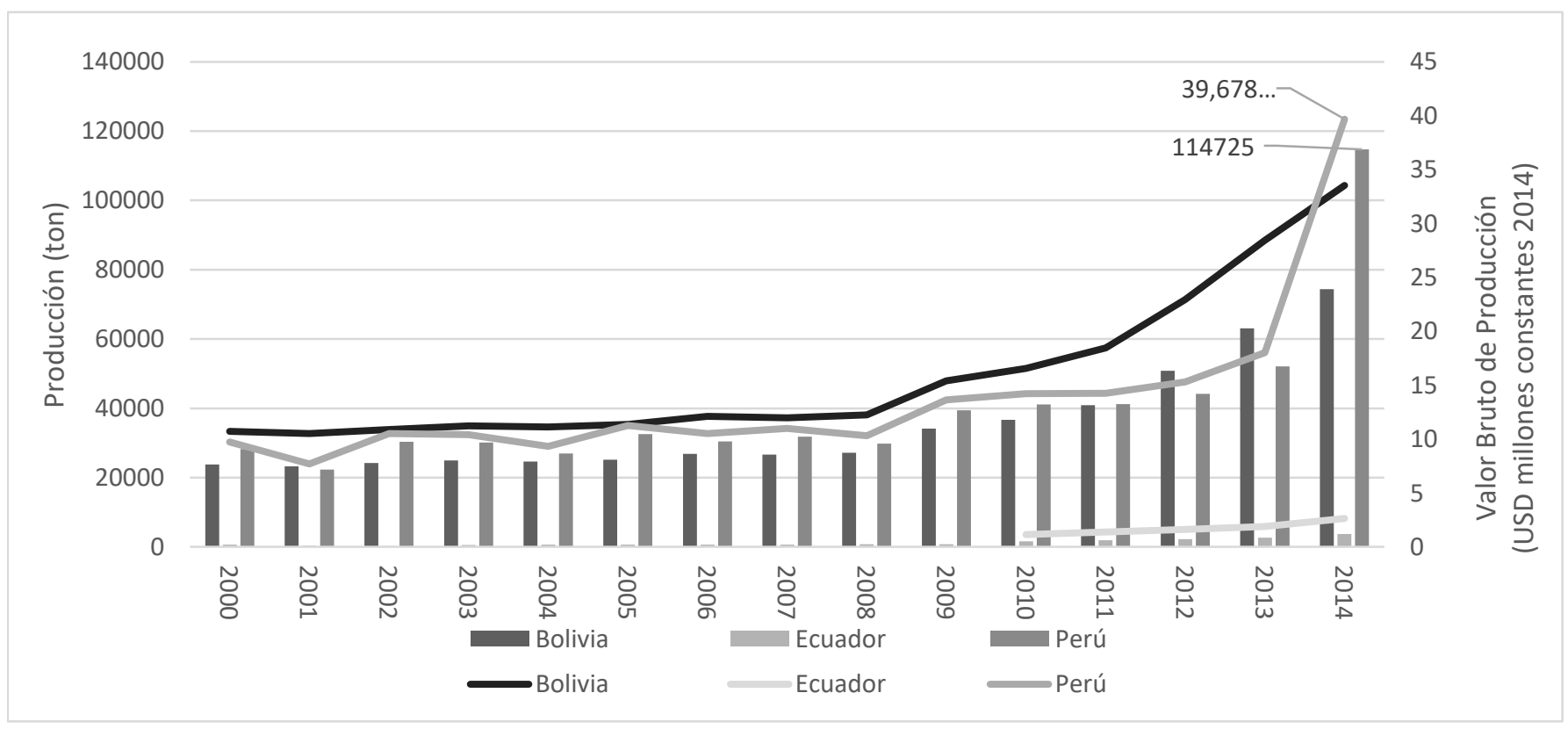

Fuente: Elaboración propia con información de FAOSTAT (2017) 
ISSN: 1794-9920 Enero - Abril de 2017

Volumen 17 Número 2, Año 2017 Págs. 157 - 173

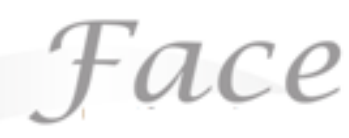

Finalmente, en términos de precios al productor (ver gráfico 4), Bolivia sigue teniendo alguna ventaja que se incrementó de manera particular en 2010 y desde entonces ha sido creciente.

\section{Gráfico N ${ }^{\circ} 4$}

Precios al productor (Bolivia y Perú)

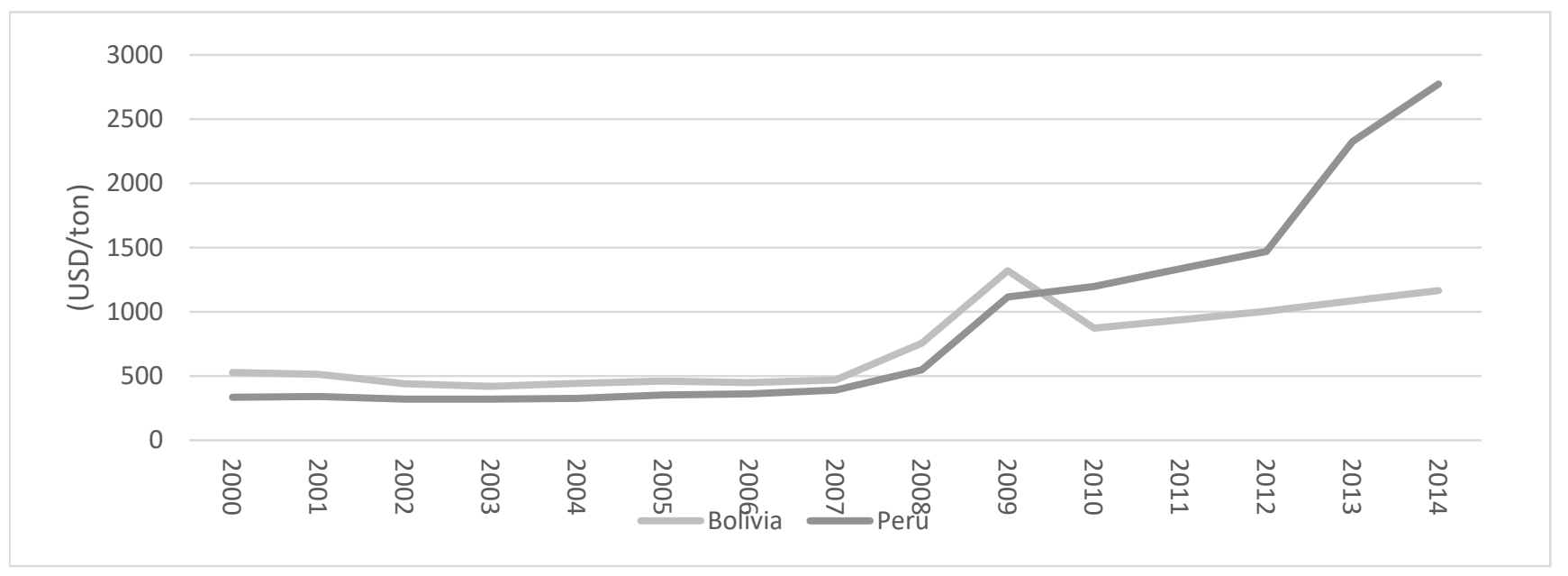

Fuente: Elaboración propia con información de FAOSTAT (2017)

En cuanto al comercio mundial, la quinua ha experimentado un crecimiento significativo en el pasado reciente, sobrepasando los 460 millones de dólares en 2014, y bajando posteriormente a 254 millones de dólares en 2016 (ITC, 2017). A pesar de esto, las importaciones y exportaciones de quinua a nivel mundial continúan al alza: las exportaciones subieron de 64528 toneladas en 2012 a 93946 toneladas en 2016, y las importaciones pasaron de 29942 toneladas a 85608 toneladas en 2016. (ITC, 2017). Aproximadamente el 80\% de las exportaciones mundiales se originan en los países de la ALADI, en particular, en tres países andinos: Bolivia, Ecuador y Perú. Los otros exportadores importantes son Estados Unidos y ciertos países de la Unión Europea, aunque en estos dos casos gran parte de las ventas corresponden a reexportaciones (ver gráfico 5).

\section{Gráfico N⒌}

Países exportadores e importadores de quinua en 2016

Paises importadores de quinua a 2015

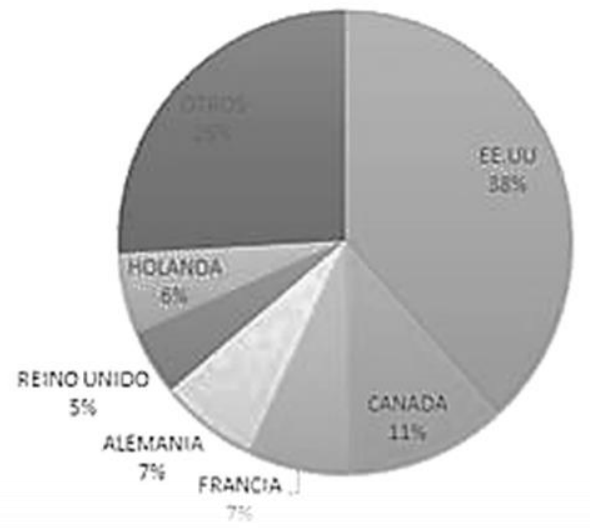

Fuente: Elaboración propia con información de TradeMap - ITC (2017)
Paises exportadores de quinua a 2016

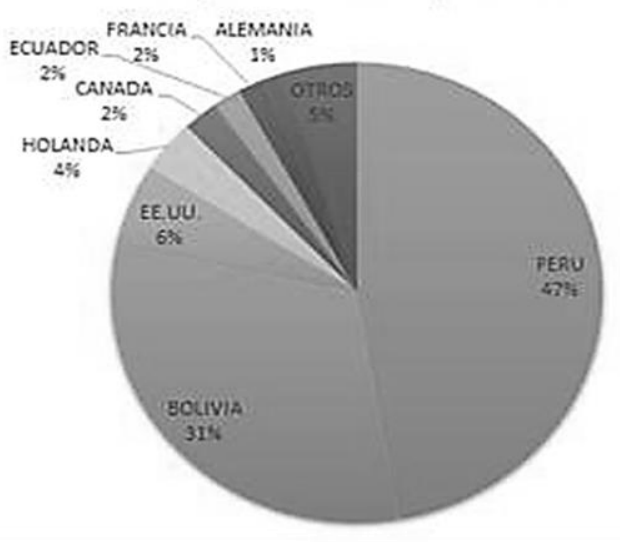


Teniendo en cuenta que el comercio de la quinua continúa en auge es importante para Colombia aprovechar la posición geográfica estratégica y los recursos con los que cuenta para ubicarse como un fuerte competidor en este mercado. Dicho lo anterior respecto a producción y comercio internacional, para poder establecer las ventajas competitivas que tienen las cadenas productivas de quinua en Bolivia, Perú y Colombia, primero se determina la conformación de la cadena de producción de quinua en general. Ésta se compone de 5 eslabones: semillas/insumos, producción primaria, transformación, comercialización y consumo. En el eslabón Insumos/semillas, se ubica todo lo pertinente al equipamiento e insumos necesarios para el cultivo de la quinua, iniciando desde la semilla (dependiendo del tipo que se vaya a producir) e incluyendo los materiales físicos (fertilizantes, maquinaria de tracción para la siembra, etc). En el eslabón Producción primaria, se distinguen las actividades propias de cultivo y cosecha de la quinua: siega, emparve, trilla y limpieza. El eslabón Transformación, incluye las actividades de pos-cosecha (desaponificación, secado) y procesamiento del grano (generación de subproductos y derivados) y el empaquetamiento. La Comercialización implica el transporte, la distribución, promoción y venta del producto. Y por último el eslabón Consumo, constituye la adquisición del producto por parte de los consumidores. Teniendo en cuenta estos 5 eslabones se realizó un análisis comparativo para la primera fase del benchmarking entre las cadenas productivas de quinua en Colombia, Perú y Bolivia, por cada eslabón que las compone (ver tabla 1).

\section{Tabla $N^{\circ} 1$.}

Comparación de las cadenas productivas por eslabón.

\begin{tabular}{|c|c|c|c|}
\hline ESLABÓN & COLOMBIA & PERÚ & BOLIVIA \\
\hline \multirow[t]{4}{*}{ INSUMOS/SEMILLAS } & $\begin{array}{l}\text { No existe un banco de } \\
\text { germoplasma establecido. }\end{array}$ & $\begin{array}{l}\text { Actualmente tiene } 8 \text { bancos de } \\
\text { germoplasma establecidos. }\end{array}$ & $\begin{array}{l}\text { Cuenta con el banco de germoplasma } \\
\text { de quinua más importante a nivel } \\
\text { mundial. }\end{array}$ \\
\hline & $\begin{array}{l}\text { Falta de caracterización de la } \\
\text { semilla. }\end{array}$ & Acceso limitado a semilla de calidad. & $\begin{array}{l}\text { Desarrollo de protocolos para la } \\
\text { caracterización de la semilla. }\end{array}$ \\
\hline & $\begin{array}{l}\text { Pocas áreas destinadas al cultivo } \\
(2550 \mathrm{Ha}) \text {. Rendimiento promedio } \\
\text { de } 1500 \mathrm{Kg} / \mathrm{Ha}\end{array}$ & $\begin{array}{l}\text { Área de cultivo de aprox. } 68000 \mathrm{Ha} \text {. } \\
\text { Rendimiento promedio de } 1500 \\
\mathrm{Kg} / \mathrm{Ha} / \mathrm{año}\end{array}$ & $\begin{array}{l}\text { Área de cultivo superior a las } 100000 \\
\mathrm{Ha} \text {. Rendimiento promedio de } 600 \\
\mathrm{Kg} / \mathrm{Ha} / \mathrm{año}\end{array}$ \\
\hline & $\begin{array}{l}\text { Áreas sembradas en pequeñas } \\
\text { parcelas de media hectárea. Hay } \\
\text { poca diversidad de la semilla (se } \\
\text { cultiva principalmente ecotipo } \\
\text { Nariño) }\end{array}$ & $\begin{array}{l}\text { El cultivo se realiza mayoritariamente } \\
\text { en parcelas de } 3 \text { ha. Se cultivan } \\
\text { alrededor de } 20 \text { variedades de } \\
\text { quinua. }\end{array}$ & $\begin{array}{l}\text { La siembra se realiza } \\
\text { mayoritariamente en parcelas } \\
\text { menores de } 500 \mathrm{~m} 2 \text {. Se cultivan más } \\
\text { de } 20 \text { variedades que conforman el } \\
\text { complejo de Quinua Real }\end{array}$ \\
\hline PRODUCCIÓN PRIMARIA & $\begin{array}{l}\text { Poca formación en el manejo del } \\
\text { cultivo y pos-cosecha. }\end{array}$ & $\begin{array}{l}\text { Acceso limitado a maquinaria para } \\
\text { cosecha y pos-cosecha con respecto } \\
\text { al área cultivada. }\end{array}$ & $\begin{array}{l}\text { Acceso relativamente limitado a la } \\
\text { maquinaria para el cultivo y pos- } \\
\text { cosecha }\end{array}$ \\
\hline \multirow[t]{2}{*}{ TRANSFORMACIÓN } & $\begin{array}{l}\text { Poca armonización con los entes } \\
\text { reguladores de calidad y } \\
\text { fitosanitarios. }\end{array}$ & $\begin{array}{l}\text { Poca sensibilización a los procesos } \\
\text { de certificación de calidad. }\end{array}$ & $\begin{array}{l}\text { El procesamiento mecanizado se ha } \\
\text { extendido. }\end{array}$ \\
\hline & $\begin{array}{l}\text { Acceso limitado a maquinaria de } \\
\text { procesamiento. Poca asistencia } \\
\text { técnica. }\end{array}$ & Poca asistencia técnica. & $\begin{array}{l}\text { Aún hay presencia de procesamiento } \\
\text { por métodos tradicionales. }\end{array}$ \\
\hline \multirow[t]{2}{*}{ COMERCIALIZACIÓN } & Altas cargas tributarias. & \multirow{2}{*}{$\begin{array}{l}\text { Presencia de muchos intermediarios } \\
\text { en los canales de comercialización } \\
\text { (internos y externos) }\end{array}$} & \multirow[t]{2}{*}{$\begin{array}{l}\text { Diversos canales de comercialización } \\
\text { (interno y externo). }\end{array}$} \\
\hline & $\begin{array}{l}\text { Mayoritariamente } \\
\text { externos. }\end{array}$ & & \\
\hline
\end{tabular}




\begin{tabular}{|c|l|l|l|}
\hline & $\begin{array}{l}\text { Exportaciones principalmente a } \\
\text { USA y Canadá }\end{array}$ & $\begin{array}{l}\text { Exportaciones principalmente a USA, } \\
\text { Francia, Alemania e Italia }\end{array}$ & $\begin{array}{l}\text { Exportaciones a USA, Canadá y } \\
\text { ciertos países de Europa }\end{array}$ \\
\hline CONSUMO & $\begin{array}{l}\text { Desconocimiento del producto } \\
\text { internamente. Bajo fomento del } \\
\text { consumo interno. }\end{array}$ & $\begin{array}{l}\text { Hay una tendencia al aumento del } \\
\text { consumo interno, específicamente en } \\
\text { las zonas urbanas. }\end{array}$ & Bajo consumo interno. \\
\hline
\end{tabular}

Fuente: elaboración propia con información de FAO / ALADI (2014) - IICA (2015) - Jägger (2015a y b) - Gobernación del Cauca (2016) CAF/CDI/CLACDS (2001) / Ministerio de Agricultura y Riego del Perú (2017) / Risi, Rojas y Pacheco (2015) y entrevistas.

A partir de esta comparación se puede determinar que teniendo en cuenta la caracterización de los eslabones, la cadena productiva de Colombia presenta "cuellos de botella" considerables que deben ser mejorados para poder elevar el nivel de competitividad del país en el mercado de la quinua. Con respecto a las ventajas y desventajas que cada de una de las cadenas productivas de los países seleccionados se realiza un estudio comparativo aplicando la técnica del benchmarking y basándose en los determinantes del diamante de la competitividad de Michael Porter. En la tabla 2 se pueden observar los resultados.

Tabla $\mathrm{N}^{\circ} 2$.

Benchmarking de la cadena productiva quinua de Perú, Bolivia y Colombia con base en los aspectos del diamante de competitividad de Michael Porter.

\begin{tabular}{|c|c|c|c|}
\hline DETERMINANTE & BOLIVIA & PERU & COLOMBIA \\
\hline \multirow{5}{*}{$\begin{array}{l}\text { FACTORES } \\
\text { PRODUCCIÓN }\end{array}$} & $\begin{array}{l}2^{\circ} \text { productor con reconocimiento a nivel } \\
\text { mundial. Producto mayormente orgánico }\end{array}$ & $\begin{array}{l}\text { Actual productor líder con reconocimiento } \\
\text { a nivel mundial }\end{array}$ & $\begin{array}{l}\text { No posiciona en el ranking de países } \\
\text { productores. No hay reconocimiento } \\
\text { del producto }\end{array}$ \\
\hline & $\begin{array}{l}\text { Gran área de cultivo con producción de } \\
83.603 \text { ton/año ; bajo rendimiento } \mathrm{kg} / \mathrm{ha} \text {, } \\
\text { una sola cosecha al año }\end{array}$ & $\begin{array}{l}\text { Gran área de cultivo, más de } 60.000 \text { ha, } \\
\text { con producción de } 114.300 \text { ton/año, una } \\
\text { sola cosecha de alto rendimiento. }\end{array}$ & $\begin{array}{l}\text { Poca área de cultivo menos de } 3.000 \text { ha } \\
\text { con producción de } 4.781 \text { ton } / \text { año, alto } \\
\text { rendimiento } 1.500 \mathrm{~kg} / \mathrm{ha} \text {. Dos cosechas } \\
\text { anuales }\end{array}$ \\
\hline & $\begin{array}{l}\text { Ubicación geográfica privilegiada, cultivo } \\
\text { adaptable a diferentes condiciones }\end{array}$ & $\begin{array}{l}\text { Ubicación geográfica privilegiada, cultivo } \\
\text { adaptable a diferentes condiciones }\end{array}$ & $\begin{array}{l}\text { Ubicación geográfica privilegiada } \\
\text { cultivo adaptable a diferentes } \\
\text { condiciones }\end{array}$ \\
\hline & $\begin{array}{l}\text { Diversidad de Germoplasma y actividades } \\
\text { de investigación }\end{array}$ & $\begin{array}{l}\text { Diversidad de Germoplasma y actividades } \\
\text { de investigación }\end{array}$ & $\begin{array}{l}\text { No hay tipificación de la semilla usada, } \\
\text { no hay banco de germoplasma. }\end{array}$ \\
\hline & $\begin{array}{l}\text { Presenta técnicas ancestrales de cosecha } \\
\text { y pos cosecha, con poca formación en } \\
\text { prácticas agrícolas y maquinaria } \\
\text { tecnificada }\end{array}$ & $\begin{array}{l}\text { Aún presenta técnicas ancestrales de } \\
\text { cosecha y pos cosecha, acceso a } \\
\text { maquinaria tecnificada y bajos costos de } \\
\text { producción. }\end{array}$ & $\begin{array}{l}\text { Técnicas ancestrales de cosecha y pos } \\
\text { cosecha, bajo acceso a maquinaria } \\
\text { tecnificada }\end{array}$ \\
\hline \multirow{4}{*}{$\begin{array}{l}\text { CONDICIONES DE LA } \\
\text { DEMANDA }\end{array}$} & $\begin{array}{l}\text { Conocimiento a nivel interno del producto. } \\
\text { Bajo consumo del mismo en la zona } \\
\text { urbana }\end{array}$ & $\begin{array}{l}\text { Conocimiento del producto a nivel interno, } \\
\text { consumo creciente en la zona urbana }\end{array}$ & $\begin{array}{l}\text { No existe promoción del consumo de } \\
\text { producto, Consumo sectorizado. }\end{array}$ \\
\hline & $\begin{array}{l}\text { Incremento importante de la demanda } \\
\text { internacional de productos orgánicos. }\end{array}$ & $\begin{array}{l}\text { Incremento importante de la demanda } \\
\text { internacional de productos orgánicos }\end{array}$ & $\begin{array}{l}\text { Incremento importante de la demanda } \\
\text { internacional de productos orgánicos }\end{array}$ \\
\hline & $\begin{array}{l}\text { Informalidad en el comercio internacional, } \\
\text { no hay distinción del producto orgánico y } \\
\text { certificado. No existe demanda de } \\
\text { producto con valor agregado }\end{array}$ & $\begin{array}{l}\text { Vías de comercialización establecidas. } \\
\text { Producto sin valor agregado. No hay } \\
\text { marcas propias }\end{array}$ & $\begin{array}{l}\text { Caracterizado por su sabor dulce no es } \\
\text { altamente demandado. }\end{array}$ \\
\hline & Alta oferta de producción & $\begin{array}{l}\text { Alta producción y bajo costos en la } \\
\text { exportación }\end{array}$ & Vías de comercialización incipientes. \\
\hline
\end{tabular}


Jahir Lombana - Yasser Amashta - Carolina Correa - Maria-Carolina Rodríguez

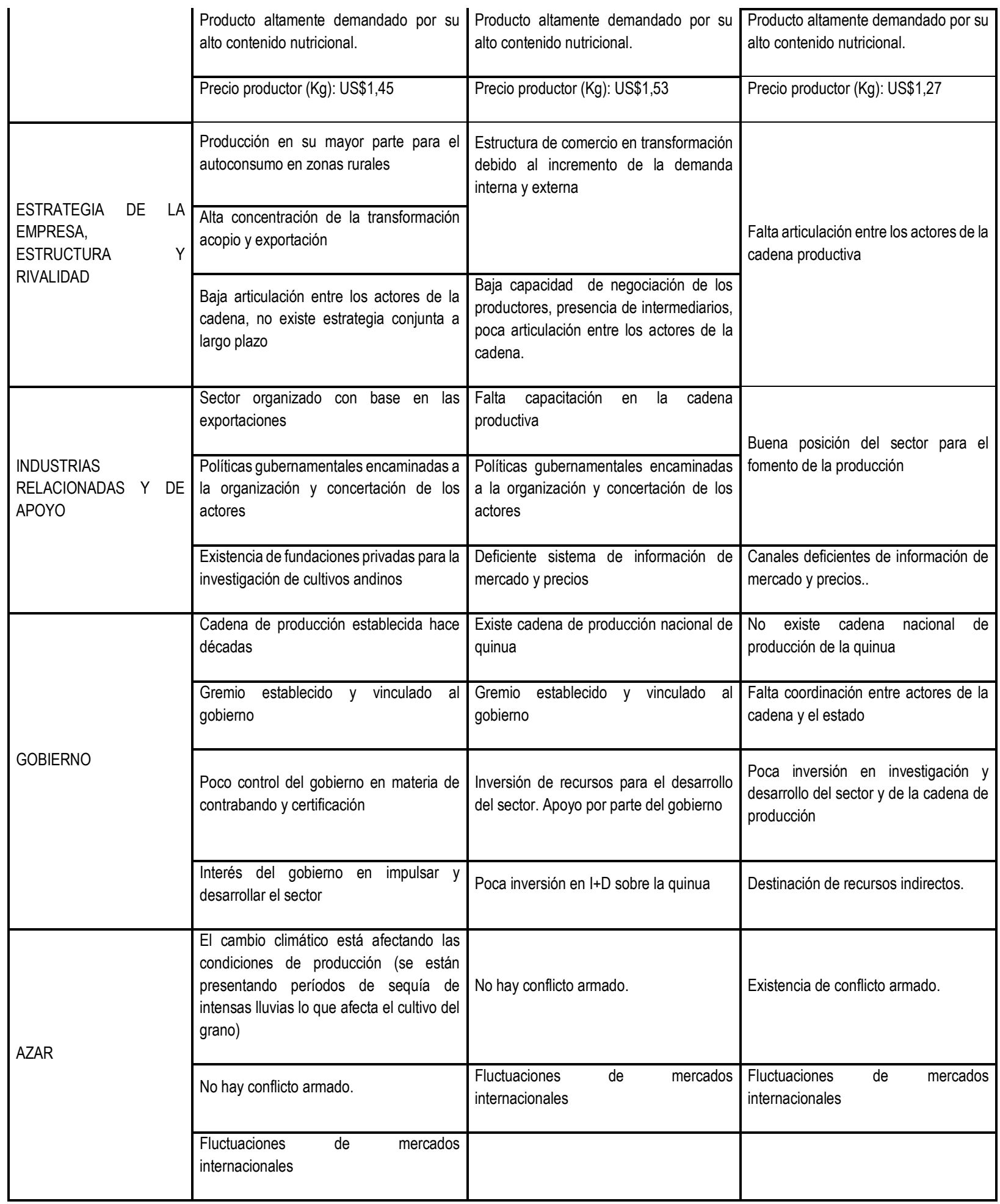

Fuente: elaboración propia con información de FAO / ALADI (2014) - IICA (2015) - Jägger (2015a y b) - Gobernación del Cauca (2016) - CAF/CDI/CLACDS (2001) / Ministerio de Agricultura y Riego del Perú (2017) / Risi, Rojas y Pacheco (2015) y entrevistas. 
Luego de la revisión recolectada y el análisis comparativo encontramos que el mercado de la quinua está en ascenso en los tres países. Por ser países andinos, se encuentran en una privilegiada posición para el cultivo del grano (aunque las tasas de rendimiento son diferentes para cada uno), sin embargo Colombia por la mínima cantidad de hectáreas cultivadas se encuentra rezagada en este determinante. Por otra parte, mientras que en los tres países se conservan técnicas ancestrales para los procesos de cosecha y pos cosecha, en Perú hay un mayor acceso a maquinaria tecnificada y cabe anotar que tanto Perú como Bolivia poseen bancos de Germoplasma con la respectiva tipificación, mejoramiento y conservación del grano. Bolivia es el país en el que el peso del sector agrícola en su PIB es mayor (13\%), mientras que en Perú tiene un peso de $7,7 \%$ y en Colombia tiene un peso de $6,80 \%$. Bolivia es un país fuertemente agrícola, lo que explica la gran extensión de hectáreas destinadas a cultivos como la quinua.

La demanda de quinua ha crecido a nivel mundial y esto ha jalonado el crecimiento de la oferta productiva en los tres países, más el interés del mercado internacional está radicalizado en el grano preferiblemente de procesos orgánicos, lo que no ha permitido a los grandes productores comercializar a gran escala productos con valor agregado y de marcas propias. Los precios varían de país a país destacando Colombia con un menor precio al productor (US $\$ 1,27 \mathrm{~kg}$ ) pero esto no hace establece una brecha entre el precio de Perú y Bolivia. La existencia de varios intermediarios y no de una cadena productiva sólida con fines comunes hace finalmente el precio FOB colombiano el más caro para el mercado (US\$5,42kg) lo que le resta a el país la competitividad frente a Perú y Bolivia.

Las industrias de apoyo y los sistemas de difusión y precios son deficientes para los tres países analizados y en Colombia se ve muy marcado en la desinformación acerca del producto y los mínimos esfuerzos realizados por la fomentación del consumo del mismo. Bolivia y Perú por su parte presentan consumos internos, en el área rural y urbana respectivamente. Es importante anotar que los actores de la cadena se encuentran desarticulados en los tres países pero tanto en Bolivia como en Perú, existen instituciones a nivel privado y gubernamental que promueven espacios para el desarrollo de planes de mejoramiento a largo plazo del sector.
La influencia del Gobierno es significativa en los 3 países en la creación de políticas que fomenten e incentiven el cultivo del grano y en la distribución de los recursos necesarios para la producción. En el caso de Bolivia y Perú, los gobiernos de dichos países han realizado más inversiones que el gobierno colombiano, sin embargo se requieren aún más recursos destinados para poder alcanzar la tecnificación de la producción y el acceso a insumos de calidad, al igual que regular los procesos de certificación de calidad y ejercer más control en los casos de contrabando. Para el gobierno Colombiano se torna un poco más difícil realizar inversiones grandes, puesto que debido a la presencia del conflicto interno, muchos de los recursos son priorizados a la resolución de éste.

Eventos externos influyen sobre la producción del grano, como lo es el cambio climático que viene afectando las condiciones en los territorios de cultivo. Los cambios del mercado en los que se desenvuelven los países también tienen un impacto directo en el sector. Las alteraciones en las tasas de cambio influyen directamente en la comercialización del producto. El precio del dólar en especial tiene un efecto directo en los costos y utilidades para los productores y comercializadores.

En Colombia, el cultivo de la quinua es un proceso relativamente nuevo y aún se encuentra en la etapa de consolidación. Para llegar a alcanzar una posición dentro del mercado mundial de la quinua, es necesario destinar recursos (económicos y humanos) para la creación y el desarrollo de programas diseñados para el establecimiento de una cadena productiva sólida y eficiente.

Teniendo en cuenta las comparaciones obtenidas del ejercicio del benchmarking y basándose en los determinantes del diamante de competitividad de Porter, resultaron los siguientes factores críticos de éxito para el mejoramiento del nivel de competitividad de la cadena productiva de quinua en Colombia. 
Tabla $N^{\circ} 3$.

Factores críticos de éxito para Colombia en la cadena productiva de quinua

\begin{tabular}{|c|c|}
\hline $\begin{array}{l}\text { ASPECTO } \\
\text { ANALIZADO }\end{array}$ & FACTORES CRÍTICOS DE ÉXITO \\
\hline $\begin{array}{l}\text { FACTORES DE } \\
\text { PRODUCCIÓN }\end{array}$ & $\begin{array}{l}\text { Aumento de las áreas de cultivo, caracterización de la } \\
\text { semilla, facilitación de acceso a maquinaria técnica, índices } \\
\text { de rendimiento de producción }\end{array}$ \\
\hline $\begin{array}{c}\text { CONDICIONES DE LA } \\
\text { DEMANDA }\end{array}$ & Aumento de la oferta del producto, promoción del consumo \\
\hline $\begin{array}{l}\text { INDUSTRIAS } \\
\text { RELACIONADAS Y } \\
\text { DE APOYO }\end{array}$ & $\begin{array}{l}\text { Apertura de espacios de discusión y comunicación, } \\
\text { facilitación del flujo de la información, desarrollo de } \\
\text { estrategias de marketing }\end{array}$ \\
\hline $\begin{array}{l}\text { ESTRATEGIA, } \\
\text { ESTRUCTURA DE } \\
\text { EMPRESA Y } \\
\text { RIVALIDAD }\end{array}$ & $\begin{array}{l}\text { Apoyo a las empresas incipientes en su conformación y } \\
\text { estructura, acompañamiento a empresas en expansión }\end{array}$ \\
\hline GOBIERNO & $\begin{array}{l}\text { Consolidación y organización de la cadena de producción } \\
\text { nacional, fomento de inversión e incentivos para el cultivo, } \\
\text { formación del recurso humano, inversión sostenida de } \\
\text { capital, promulgación de políticas regulatorias }\end{array}$ \\
\hline
\end{tabular}

Fuente: elaboración propia

Con respecto a los factores de producción, se encuentra que una de las debilidades en Colombia es el poco acceso a la tecnología requerida para una producción de la quinua. Por lo tanto se debe empezar por tipificar el ecotipo del grano que se utiliza y crear un banco de germoplasma para impulsar los estudios de diversidad genética de la semilla. Luego continuar con el proceso de certificación de calidad del producto; desarrollar programas intensivos de formación de la fuerza laboral (instruir a los agricultores acerca de las diferentes técnicas ancestrales y modernas para el cultivo del grano) y facilitar la adquisición de la maquinaria requerida para los procesos de cosecha y pos cosecha.

Para todo esto es necesario una fuerte presencia del sector académico, especialmente de las áreas de ingenierías para el diseño, desarrollo y aplicación de estrategias y tecnologías para la mejoría de la producción. Actualmente Colombia ha alcanzado un hito histórico con la firma de los Acuerdos de Paz el pasado noviembre de 2016. Si bien es

cierto que esta etapa de posconflicto es frágil aún, este acuerdo pone fin a más 50 años de guerra entre el estado y la guerrilla de las FARC, que ha sido uno de los más cruentos y devastadores grupos insurgentes en la historia del país. Esto le da un nuevo aire al panorama nacional, puesto que una parte de los recursos que previamente estaban siendo destinados para la lucha contra las FARC, podrían en teoría ser dedicados al reclamo de tierras previamente ocupadas por la guerrilla para la plantación de cultivos ilícitos, y darle un nuevo impulso al sector agrícola. Aunque es cierto que otro tipo de cultivos tradicionales tendrían cierta "prioridad" al momento de la destinación de estos recursos, las características del cultivo de la quinua y su producción (alto rendimiento, adaptabilidad del grano, etc.) han logrado despertar el interés del gobierno nacional para continuar con el mejoramiento de la cadena productiva.

En el departamento del Cauca los planes que se están llevando a cabo para fomentar el cultivo de la quinua están siendo financiados por el Sistema General de Regalías del Departamento Nacional de Planeación, el cual "determina la distribución, objetivos, fines, administración, ejecución, control, el uso eficiente y la destinación de los ingresos provenientes de la explotación de los recursos naturales no renovables precisando las condiciones de participación de sus beneficiarios" (DNP, 2012), lo cual ha significado un desarrollo en el cultivo del grano en el Cauca. Varias empresas en Popayán se han visto beneficiadas por esta iniciativa y se ha logrado que actualmente Cauca tenga el mayor rendimiento de producción a nivel nacional con aproximadamente $1500 \mathrm{Kg} / \mathrm{Ha} / \mathrm{año}$, y sea posible realizar 2 cosechas anualmente, similar al rendimiento Peruano. (Jägger, 2015a).

No sólo es importante buscar la mejoría de la producción primaria, sino también la transformación. Se deben desarrollar líneas de productos con valor agregado con una o varias marcas propias nacionales para obtener los beneficios y utilidades dejados por la comercialización de éstos, puesto que una gran parte del mercado actual de la quinua se caracteriza por la importación-transformaciónreexportación por países tales como E.E.U.U. y algunos países miembros de la Unión Europea. Es importante que el aspecto regulatorio entre en juego. Se necesita una fuerte 
décadas. Además, las áreas destinadas para el cultivo de la

regulación del producto de contrabando, además de políticas gubernamentales que garanticen la calidad del producto a través de certificaciones fitosanitarias y que se logre una diferenciación del producto orgánico, que es el más apetecido mundialmente.

En Colombia la quinua es un producto nuevo y poco reconocido a nivel nacional. Esto hace que el consumo interno sea bajo, y a su vez esto influye en la producción de la quinua. Si no se conoce y no se consume es mucho más difícil incrementar los niveles de producción. Actualmente el mercado de la quinua en el país está restringido a pequeños grupos focales interesados en los productos orgánicos y naturistas. Es necesario que se generen programas con una plataforma más grande que le permite al ciudadano colombiano promedio conocer acerca de este producto y elevar los niveles de consumo dentro del país, que no implica solamente un desarrollo económico para el gremio, sino también implica un mejoramiento en los hábitos alimenticios de la población nacional. Estrategias de marketing intensivo se vuelven indispensables para lograr ubicar el producto nacional de quinua al alcance de la población nacional.

Un punto importante a destacar seria que Colombia logre posicionarse como productor de quinua en el mercado mundial, puesto que investigaciones preliminares que se están realizando en países como Italia y países en África han arrojado resultados positivos para el inicio del cultivo del grano en sus territorios, lo que implicaría la aparición de nuevos competidores. Se deben aprovechar las ventajas que tiene Colombia en el ámbito de los recursos naturales y explotarlos antes de que la demanda de quinua que el momento está en auge y le está representando a Perú y Bolivia ingresos anuales considerables, decaiga y se torne más difícil lograr un nivel competitivo con países con una capacidad mayor de otros recursos.

\section{CONCLUSIONES:}

Al realizar el análisis comparativo del estado de la cadena de producción de quinua en Bolivia y Perú en relación a Colombia, se pudo establecer un panorama claro de las ventajas y desventajas presentadas en la cadena nacional. Se encontró que Perú y Bolivia tienen el liderazgo mundial en producción y comercialización de la quinua, ocupando el primer y segundo lugar respectivamente y que tales actividades preceden a la producción colombiana en quinua en Perú y Bolivia son extensas y ambos países tienen bancos de germoplasma con gran cantidad de accesiones. Adicionalmente los gobiernos peruano y boliviano realizan inversiones en investigación, tecnología, financiamiento y promoción del cultivo, lo que impulsa aún más la actividad productora. Esto resulta en el establecimiento de incipientes empresas generadoras de valor agregado al producto.

A pesar de lo anterior también se encontró que las cadenas de Perú y Bolivia presentan falencias similares a las de Colombia: aún hay poco acceso a tecnologías innovadoras, conservan técnicas de cultivo ancestrales, falta de formación de la mano de obra, la articulación de los actores de los eslabones de la cadena no es óptima y siguen siendo principalmente exportadores de grano. Fuera de estos factores mencionados, en Colombia existen otros factores de carácter socio-político que también afectan la destinación de grandes recursos a este nuevo producto, como es el conflicto interno que se vive en Colombia desde hace más de 50 años. Sin embargo, se espera que ahora que se ha dado el primer paso para el final del conflicto con la desmovilización de la guerrilla de las FARC, se reestructuren los planes de inversión y el producto de la quinua se vea beneficiado en estos.

Colombia presenta ventajas que pueden ser potencializadas con el fin de mejorar su competitividad. Las tierras óptimas para el cultivo no son de difícil acceso como el caso de Bolivia donde la siembra se hace en sitios de gran altura y difíciles condiciones climáticas. Tiene un alto rendimiento de la producción, que solamente con los resultados del departamento del Cauca logra prácticamente doblar el rendimiento de la producción en Perú y Bolivia, bajos costos de producción, y un interés considerable por parte de los actores de la cadena para su consolidación.

Si se busca mejorar el nivel de competitividad de la quinua en Colombia, es prioritario un apoyo integral del gobierno en temas como: el liderazgo en consolidación de la cadena y gremio; inversión en investigación y tecnología para los eslabones de insumos, producción y transformación; promoción del cultivo a través de incentivos y financiación; inversiones en capacitación de mano de obra e infraestructura de logística; apoyo a las empresas incipientes que generen valor agregado al producto; $y$ 
creación de un marco regulatorio que garanticen el adecuado estado fitosanitario y la certificación de calidad.

Actualmente, la demanda de quinua en el mundo está en su apogeo y de acuerdo a las circunstancias internas del país, es el momento justo para que Colombia le apueste a la producción de quinua (acciones para finiquitar el conflicto social y la firma de TLCs con diversos países), puesto que esto significaría un beneficio tanto para la nación con el crecimiento y desarrollo económicos que generaría, como para el mundo al contribuir a la producción de un grano que es considerado como un alimento capaz de combatir los niveles de hambre y garantizar la seguridad alimentaria.

\section{REFERENCIAS:}

Bhargava A. y Ohri D. (2014). Quinoa in the Indian subcontinent. En: Bazile D. Bertero H.D. \& Nieto C. (eds.). Estado del arte de la quinua en el mundo en 2013. Rome: FAO-CIRAD, pp. 511-523

Boxwell, R. (1994). Benchmarking para Competir con Ventaja. S.A. Mcgraw-Hill / Interamericana de España.

Camp, R. C. (1989). Benchmarking - The Search for Industry Best Practices That Lead to Superior Performance. Milwaukee, WI: ASQC Quality Press.

Cely Torres, L. A. y Ducon-Salas, J. C. (2015). Posibilidades en el comercio internacional de la quinua: un análisis desde la perspectiva de la competitividad. Equidad \& Desarrollo, (24), 119-137. Recuperado de https://revistas.lasalle.edu.co/index.php/ ed/article/view/3683

Cifuentes W., Pérez, M. y Gil-Casares M. (2011). Metodología de análisis de cadenas productivas bajo el enfoque de cadenas de valor. Fundación CODESPA. Recuperado de http://www.codespa.org/blog/publicacio nes-notas-tecnicas/metodologia-deanalisis-de-cadenas-productivas-bajo-unenfoque-de-cadenas-de-valor/
Corporación Andina de Fomento (CAF); Centro para el Desarrollo Internacional - Universidad de Harvard (CID); Centro Latinoamericano para la Competitividad y el Desarrollo Sostenible (CLACDS - INCAE). (2001). Caracterización y Análisis de la Competitividad de la Quinua en Bolivia. La Paz. Recuperado de: https://www.academia.edu/5059499/CA RACTERIZACI\%C3\%93N_Y_ANALISIS_DE_L A_COMPETITIVIDAD_DE_LA_QUINOA_EN _BOLIVIA

Coulibaly A., Sangaré A., Konate M., Traoré S., Ruiz B, Martinez E.A., Zurita A., Antognoni F., Biondi S., Maldonado S., Léon P., Bazile D. (2014). Evaluación de quinoa (Chenopodium quinoa Willd.) y adaptación a las condiciones agroclimáticas de Mali, África Oeste: Un ejemplo de colaboración Sur-Norte-Sur. En: Bazile D. Bertero H.D. \& Nieto C. (eds.). Estado del arte de la quinua en el mundo en 2013. Rome : FAO-CIRAD, pp. 635-646.

Dunning, J. H. (1993). Internationalizing Porter's diamond. Management International Review, (33), pp.7-15.

Fernandez, P., Mccarthy I.P. y Rakotobe-Joel T. (2001) An evolutionary approach to benchmarking. Benchmarking: An International Journal, 8 (4), pp. 281-305.

Food and Agriculture Organization for the United Nations - FAO y Asociación Latinoamericana de Integración - ALADI. (2014). Tendencias y Perspectivas del Comercio Internacional de Quinua. Santiago: FAO. Recuperado de http://www.fao.org/publications/card/es /c/90ce9b3a-7f09-5d64-b4ef6990d4958dfb/

Food and Agriculture Organization for the United Nations - FAO. (2017). FAOStat. Estadísticas Agrícolas y del Sector Alimentario. Recuperado de: http://www.fao.org/faostat/en/

Food and Agriculture Organization for the United Nations - FAO. (2011). Quinua: Cultivo milenario para contribuir a la seguridad alimentaria mundial. FAO. Recuperado de: http://www.fao.org/docrep/017/aq287s/ aq287s.pdf 
Food and Agriculture Organization for the United Nations - FAO. (2013). Quinua: Operaciones de Poscosecha. AGSI/FAO. Recuperado de http://www.fao.org/3/a-ar364s.pdf

Food and Agriculture Organization for the United Nations - FAO. (2014). State of the art report on quinua around the world in 2013.Santiago: FAO. Recuperado de http://www.fao.org/3/ai4042s.pdf

Gobernación del Cauca - República de Colombia. (2016). Quinua: Una alternativa de crecimiento económico para los agricultores del Cauca. Recuperado

de http://www.cauca.gov.co/noticias/quinu a-una-alternativa-de-crecimientoeconomico-para-los-agricultores-delcauca

Gomes de Castro, A.M., Valle-Lima S. y Freitas A. (1998). Módulo de Capacitação em Prospecção Tecnológica de Cadeias Produtivas. Embrapa/DPD, Brasília.

Gomes de Castro, A.M., Valle-Lima S. y Pedroso C. (2002). Cadena productiva: Marco conceptual para apoyar la prospección tecnológica Revista Espacios. 23(2) Recuperado de http://www.revistaespacios.com/a02v23 n02/02230211.html

Gurutze, M. y Ochoa, C. (2005). Una Revisión Teórica de la Herramienta de Benchmarking Revista de Dirección y Administración de Empresas. 12, pp. 73-103

Hoskisson, R. E., Eden, L., Lau, C. M. y Wright, M. (2000). Strategy in emerging economies. Academy of Management Journal, (43), pp. 249-67.

Instituto Interamericano de Cooperación para la Agricultura - IICA. (2015). El Mercado y La Producción de Quinua en el Perú. Lima. Recuperado de http://www.iica.int/sites/default/files/pu blications/files/2016/b3857e.pdf

International Trade Centre - ITC. (2017). Trade Map. Trade statistics for international business development. Recuperado de: http://www.trademap.org/
Jacobsen, S.-E. (1997). Adaptation of quinua (Chenopodium quinua) to Northern European agriculture: studies on developmental pattern. Euphytica 96, pp.41-48.

Jägger, M. (2015a). La Quinua a la conquista del mundo: La quinua en Colombia en el año 2020. Centro Internacional de Agricultura Tropical (CIAT); Corporación Colombiana de Investigación Agropecuaria (CORPOICA), $1 p$

Jägger, M. (2015b). El cultivo de la quinua en Colombia y sus perspectivas futuras. Cali.

Krugman, P. (1994) Competitiveness: A Dangerous Obsession. Foreing Affairs March/April 28-44.

Krugman, P. (2005). El internacionalismo moderno. la economía internacional y las mentiras de la competitividad, Edit. Critica, colección Biblioteca de Bolsillo, $2^{\mathrm{a}}$ edición, Barcelona. $171 \mathrm{p}$.

Kyrö, P. (2003). Revising the concept and forms of benchmarking, Benchmarking: An International Journal, 10 (3), pp. 210-225.

Lombana J. (2006). Competitiveness and Trade Policy Problems in Agricultural Exports :A Perspective of Producing/Exporting Countries in the Case of Banana Trade to the European Union. Dissertation for the PhD Degree in Economics at the University of Goettingen.

Lombana, J. y Rozas S. (2009). Marco analítico de la competitividad. Fundamentos para el estudio de la competitividad regional. Pensamiento \& gestión, 26, pp. 1-38

McNair, C. y Leibfried, K. (1992). Benchmarking: A Tool for Continuous Improvement. Essex Junction, VT: Omneo Oliver Wright Publications, Inc.

Ministerio de Agricultura y Riego del Perú (2015) El Perú es el principal productor y exportador de quinua en el mundo. Recuperado de http://minagri.gob.pe/portal/noticiasanteriores/notas-2015/12000-el-peru-esel-principal-productor-y-exportador-dequinua-en-el-mundo

Ministerio de Agricultura y Riego del Perú (2017) La Quinua: Producción y Comercio del Perú. Perfil Técnico. Recuperado de http://www.minagri.gob.pe/portal/analis 
is-economico/analisis-

2017?download=10867: quinua-comercioy-produccion-del-peru-2017

Montoya, L., Martínez, L., \& Peralta, J. (2005). Análisis de variables estratégicas para la conformación de una cadena productiva de quinua en Colombia. INNOVAR Revista de ciencias administrativas y sociales. 15 (25), pp. 103-119 Recuperado en http://www.scielo.org.co/pdf/inno/v15n 25/v15n25a07.pdf

Murphy K. y Matanguihan J. (2015). Quinoa Cultivation for Temperate North America: Considerations and Areas for Investigation. En Peterson A. J. y Murphy K. M. (Eds). Quinoa: Improvement and Sustainable Production. John Wiley \& Sons, Inc DOI: 10.1002/9781118628041.ch10

Piva G., Brasse C. y Mehinagic E. (2013). Quinua d'Anjou: Comienzo del Sector de Quinua Francesa. En: Bazile D. Bertero H.D. \& Nieto C. (eds.). Estado del arte de la quinua en el mundo en 2013. Rome : FAO-CIRAD, pp. 534-541.

Porter, M. (1991) La Ventaja Competitiva de las Naciones. Edit. Javier Vergara, Buenos Aires, 1025 p., edición original The Competitive Advantage of Nations, The Free Press, New York. 1990

Porter, M. y Millar, V. (1985) How Information Gives You Competitive Advantage. Harvard Businees Review.

Risi, J., Rojas, W. y Pacheco M. (2015). Producción y Mercado de la Quinua en Bolivia. La Paz: Instituto Interamericano de Cooperación para la Agricultura IICA. Recuperado de http://www.iica.int/sites/default/files/pu blications/files/2015/b3763e.pdf

Rojas, P., \& Sepúlveda, S. (1999). ¿Qué es la Competitividad?. IICA. San José, Costa Rica.

Rugman, A. M. \& Verbeke, A. (1993). Foreign subsidiaries and multinational strategic manage-ment: an extension and correction of Porter's single diamond framework. Management International Review, (33), 7184.
Spendolini, Michael J. (1992). The Benchmarking Book. New York, NY: American Management Association.

Watson, G. H. (1993). Strategic Benchmarking. New York: John Wiley and Sons.

Winkel T., Bertero H.D., Bommel P., Bourliaud J., Chevarria-Lazo M., Cortes G., Gasselin P., Geerts S., Joffre R., Léger F., Martinez Avisa B., Rambal S., Rivière G., Tichit M., Tourrand J.-F., Vassas Toral A., Vacher J.J., Vieira Pak M. (2012). The sustainability of quinoa production in southern Bolivia: from misrepresentations to questionable solutions. Comments on Jacobsen (2011, J. Agron. Crop Sci. 197: 390-399). Journal of agronomy and crop science 198 (4): 314-319. doi: 10.1111/j.1439- 037X.2012.00506.x http://dx.doi.org/10.1111/j.1439037X.2012.00506.x.

Wright, M., Filatotchev, I., Hoskisson, R. E. \& Peng, M. W. (2005). Guest editor's introduction: Strategy research in emerging economies: Challenging the conventional wisdom'. Journal of Management Studies, 42 (1),1-33. 